\title{
Witnessing nonclassicality through large deviations in quantum optics
}

\author{
Dario Cilluffo $\odot,{ }^{1,2,5, *, \dagger}$ Giuseppe Buonaiuto, ${ }^{3,4,5, \dagger}$ Salvatore Lorenzo, ${ }^{1}$ G. Massimo Palma $\odot,{ }^{1,2}$ Francesco Ciccarello $\odot,{ }^{1,2}$ \\ Federico Carollo, ${ }^{3,4,5}$ and Igor Lesanovsky $3,4,5$ \\ ${ }^{1}$ Università degli Studi di Palermo, Dipartimento di Fisica e Chimica - Emilio Segrè, Via Archirafi 36, I-90123 Palermo, Italy \\ ${ }^{2}$ NEST, Istituto Nanoscienze-CNR, Piazza San Silvestro 12, 56127 Pisa, Italy \\ ${ }^{3}$ School of Physics and Astronomy, University of Nottingham, Nottingham NG7 2RD, United Kingdom \\ ${ }^{4}$ Centre for the Mathematics and Theoretical Physics of Quantum Non-Equilibrium Systems, University of Nottingham, \\ Nottingham NG7 2RD, United Kingdom \\ ${ }^{5}$ Institut für Theoretische Physik, Universität Tübingen, Auf der Morgenstelle 14, 72076 Tübingen, Germany
}

(Received 24 September 2019; accepted 20 March 2020; published 27 April 2020)

\begin{abstract}
Nonclassical correlations in quantum optics as resources for quantum computation are important in the quest for highly specialized quantum devices. Here, we put forward a methodology to witness nonclassicality of the output field from a generic quantum optical setup via the statistics of time-integrated photocurrents. Specifically, exploiting the thermodynamics of quantum trajectories, we express a known nonclassicality witness for bosonic fields fully in terms of the source master equation, thus bypassing the explicit calculation of the output light state.
\end{abstract}

DOI: 10.1103/PhysRevResearch.2.023078

\section{INTRODUCTION}

During the past decades, several platforms have been proposed for implementing efficiently quantum computing [1-3]: all of them suffer from the effect of decoherence, given by the coupling to the environment [4], which ultimately deteriorates the nonclassical properties of the systems considered. In fact, for a quantum computational scheme to outperform a classical one, one requires that at least one of its component exhibits genuinely quantum features [5]. When the environment is the electromagnetic vacuum causing photon emission, such as in dissipative optical networks [6], the statistical analysis of the output light contains the information about the dynamical features of the open quantum systems [7]. In particular, the emitted photons can be used as a resource for quantum information processing [8]. Hence, the detection and optimization of nonclassical correlations in the photons emitted by a general optical setup is of primary relevance for a variety of technological applications. In this work, we present a methodology to witness nonclassicality of the light emitted from a generic quantum optical setup via the statistics of time-integrated photocurrents. Specifically, the types of setups we consider include an open quantum system, which is the source of photons, and an optical circuit used to manipulate the emission, as shown in Fig. 1. To obtain the statistical properties of the photons arriving at the detectors we make use of the large-deviations approach [9-12]. This allows us access

\footnotetext{
*Corresponding author: dario.cilluffo@unipa.it

${ }^{\dagger}$ These authors contributed equally to the work.

Published by the American Physical Society under the terms of the Creative Commons Attribution 4.0 International license. Further distribution of this work must maintain attribution to the author(s) and the published article's title, journal citation, and DOI.
}

to the joint probability distribution of the photon counting at long times, together with relevant statistical quantities such as the fluctuations of the counting fields and corresponding cross-correlation functions. In this way a nonclassicality criterion is formulated based on the time-integrated observables of the detection [13-16]. Theoretically, this establishes, from the theoretical point of view, a natural link between the statisticalphysics approach for analyzing the output and the dynamics of open quantum systems [13], and a general class of nonclassicality measures in quantum optics. We provide simple but instructive examples, where nonclassical correlations are witnessed in different dynamical regimes of the sources and for a broad range of parameters of the components of the optical circuit. Our theoretical scheme is effective in predicting the outcomes of quantum optics experiments that make use of photon counting to witness nonclassicality [17-22].

\section{OPEN QUANTUM SYSTEMS AND LARGE DEVIATION}

Our goal is to access the statistical properties of the output light of an open quantum system emitting into $N_{L}$ different modes called $\mathcal{B}_{\mu}$, with $\mu=1, \ldots, N_{L}$. The photon-counting statistics at the detectors (see Fig. 1) provides information about the state of the open system as well as about the features of the optical circuit [7]. The counting statistics is fully characterized by the cumulants of the associated photoncounting probability distribution, which are encoded in the scaled cumulant-generating function (SCGF). Next we briefly review how to compute the SCGF in a rather general setting. The evolution of the reduced density operator of the open system $\rho$, in the Markovian approximation, is given by the well-known Lindblad master equation [23-25],

$$
\dot{\rho}=-i[\hat{H}, \rho]+\sum_{\mu=1}^{N_{L}} \mathcal{D}\left(\hat{L}_{\mu}\right) \rho \equiv \mathcal{L}[\rho],
$$




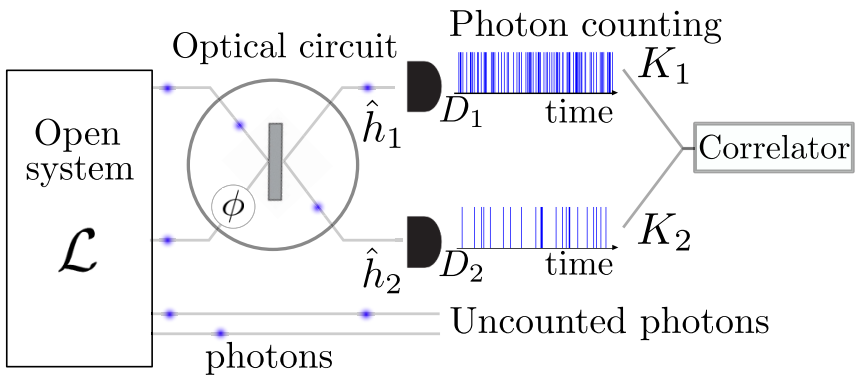

FIG. 1. Sketch of the dissipative quantum optical network. A generic quantum network is composed of a series of interconnected elements (system) emitting continuously detected radiation in the environment. The emission fields can be manipulated and transformed via a series of unitary operations in an optical circuit using beam splitters and phase shifters. The photodetectors $\left(D_{1}\right.$ and $\left.D_{2}\right)$ allow the simultaneous reconstruction of the quantum trajectories for two emission channels. Their correlation properties are studied to uncover quantum nonclassicality of radiation.

where the jump operator $\hat{L}_{\mu}$ corresponds to the interaction with the field mode $\mathcal{B}_{\mu}$ and $\mathcal{D}\left(\hat{L}_{\mu}\right)[\rho]=\sum_{\mu=1}^{N_{L}}\left[\hat{L}_{\mu} \rho \hat{L}_{\mu}^{\dagger}-\right.$ $\left.\frac{1}{2}\left\{\hat{L}_{\mu}^{\dagger} \hat{L}_{\mu}, \rho\right\}\right]$. Following a standard approach of open quantum system theory [23], we gather information about the evolution of $\rho$ by continuous monitoring of the environment.

Let us divide our jump operators into $N$ subsets, $\mathcal{J}_{i}$, each of size $n_{i}$, with $i=1, \ldots, N$, and $\sum_{i=1}^{N} n_{i}=N_{L}$ : suppose we record the occurrence of jump events due to the action of the operators in the first $M$ subsets $(M<N)$, and let $K_{m}$ be the absolute number of detected jumps in time (counting field) corresponding to each subset $\mathcal{J}_{m}$ with $m=1,2, \ldots, M$. Furthermore we assume that the action of these jump operators induces photoemission. In short notation, we define the vector $\mathbf{K}=\left(K_{1}, K_{2}, \ldots, K_{M}\right)$ to be the photon counting associated with each $\mathcal{J}_{m}$. The probability to observe $\mathbf{K}$ counts from each decay channel after a time $t$ is $P_{t}(\mathbf{K})=\operatorname{Tr}\left\{\rho^{\mathbf{K}}(t)\right\}$, where $\rho^{\mathbf{K}}(t)$ is the unnormalized reduced density operator conditioned to $\mathbf{K}$ [26]. The moment-generating function associated with $P_{t}(\mathbf{K})$ reads $Z_{t}(\mathbf{s})=\sum_{K=0}^{\infty} P_{t}(\mathbf{K}) e^{-\mathbf{s} \cdot \mathbf{K}}$, with $\mathbf{s}=\left(s_{1}, \ldots, s_{M}\right)$. Here $s_{m}$ is the conjugated field corresponding to $K_{m}$.

The outcomes of photocount experiments are timeintegrated photocurrents:

$$
\left\langle k_{i}\right\rangle=\frac{1}{t} \sum_{j=1}^{n_{i}} \operatorname{Tr}\left\{\int_{0}^{t} d \tau \hat{L}_{j}^{\dagger} \hat{L}_{j} \rho(\tau)\right\},
$$

with $i=1,2, \ldots, M$. For $t$ much greater than the typical timescale of the system $\tau_{c}$, the probability distribution associated with the photon-counting measurement takes a large deviation form [10]. Specifically, at long times the moment-generating function can be asymptotically approximated through the large-deviation theory as an exponential function of time as

$$
Z_{t}(\mathbf{s}) \sim e^{t \theta(\mathbf{s})} .
$$

This basically expresses the large-deviation principle for the moment-generating function. The analog for the count probability reads $P_{t}(\mathbf{K}) \sim e^{t \varphi(\mathbf{K} / t)}$, where $\varphi(x)=-\min _{s}\{x s+$ $\theta(s)\}$. The function $\theta(\mathbf{s})=\frac{1}{t} \ln Z_{t}(\mathbf{s})$ is the SCGF. It can be proven $[10,27]$ that this is given by the maximum real eigenvalue of the deformed superoperator

$$
\mathcal{L}_{s}[\rho]=\mathcal{L}[\rho]-\sum_{i=1}^{M}\left(1-e^{-s_{i}}\right) \sum_{\mu_{i}=0}^{n_{i}} \hat{L}_{\mu_{i}} \rho \hat{L}_{\mu_{i}}^{\dagger},
$$

which features the standard Liouvillian and the dissipator, with the jump parts corresponding to each subset $\mathcal{J}_{i}$, the latter being weighted by the factor $e^{-s_{i}}$. The cumulants of the distribution $P_{t}(\mathbf{K})$ at long times are given by the derivative of $\theta(\mathbf{s})$ at $\mathbf{s}=0$ : cumulants give direct access to the moments of the associated distribution [28].

In this work, for the sake of clarity, we consider the case $M=2$ and $n_{1}=n_{2}=1$, i.e., two distinct counting fields each associated with a single jump operator, as shown in Fig. 1. Then Eq. (4) takes the form $\mathcal{L}_{s_{1}, s_{2}}[\rho]=\mathcal{L}[\rho]-\sum_{\mu=1}^{2}(1-$ $\left.e^{-s_{\mu}}\right) \hat{L}_{\mu} \rho \hat{L}_{\mu}^{\dagger}$, and the maximum real eigenvalue of $\mathcal{L}_{s_{1}, s_{2}}$ is $\theta\left(s_{1}, s_{2}\right)=\frac{1}{t} \ln Z_{t}\left(s_{1}, s_{2}\right)$, with $Z_{t}\left(s_{1}, s_{2}\right)$ being the momentgenerating function of the probability distribution $P_{t}\left(K_{1}, K_{2}\right)$ associated with the photocount measurement described by the jump operators $\hat{L}_{\mu}$ in the long-time limit. In particular, we recover the moments of the marginal distributions $P\left(K_{1}\right)$ and $P\left(K_{2}\right)$ by setting $s_{1}=0$ or $s_{2}=0$. By exploiting the double weighting it is possible to access the correlations between the counting fields at the detectors. In particular the covariance reads

$$
\operatorname{cov}\left(k_{1}, k_{2}\right)=\left\langle k_{1} k_{2}\right\rangle-\left\langle k_{1}\right\rangle\left\langle k_{2}\right\rangle=\left.\partial_{s_{1}} \partial_{s_{2}} \theta\left(s_{1}, s_{2}\right)\right|_{s_{1}=s_{2}=0} .
$$

All other moments can be easily recovered in terms of higherorder derivatives of $\theta\left(s_{1}, s_{2}\right)$. The possibility of accessing the full statistics of the joint probability distribution, as we see in the following, allows us to make use of nonclassicality measures on the bath operators, with the idea of finding possible signatures of quantum correlations between the detection events (in the long-time limit).

\section{VOGEL'S NONCLASSICALITY CRITERION (VC)}

This criterion [29,30] gives a necessary and sufficient condition to establish whether correlations in a stationary radiation field are nonclassical or not. It consists of a rephrasing of the well-known nonclassicality criterion based on the negativity of the Glauber-Sudarshan distribution (or $\mathcal{P}$ distribution) $[31,32]$ in terms of photon-counting detection. Referring to the setup in Fig. 1, let us consider the generic bosonic operators $\hat{h}_{i}(i=1,2)$, of the two output fields, and assume they are normally ordered functions of the associated destruction and creation operators $\hat{a}_{i}$ and $\hat{a}_{i}^{\dagger}$ of each mode. A generic operator acting on the two-mode field is defined as $\hat{f}=$ $\sum_{n, m=0}^{\infty} f_{n m} \hat{h}_{1}^{\dagger n} \hat{h}_{2}^{m}$, which is a normally ordered power series of $\hat{h}_{i}$ and $\hat{h}_{i}^{\dagger}$. The expectation value of $\left\langle: \hat{f}^{\dagger} \hat{f}:\right\rangle$ reads as follows:

$$
\begin{aligned}
\left\langle: \hat{f}^{\dagger} \hat{f}:\right\rangle & =\sum_{n, m, k, l=0}^{\infty} f_{n m} f_{k l}^{*}\left|\hat{h}_{1}^{\dagger n+k} \hat{h}_{2}^{m+l}\right\rangle \\
& =\int_{\mathbb{C}} \mathcal{P}\left(\alpha_{1}, \alpha_{2}\right)\left|f\left(\alpha_{1}, \alpha_{2}\right)\right|^{2} d^{2} \alpha_{1} d^{2} \alpha_{2},
\end{aligned}
$$


(a)

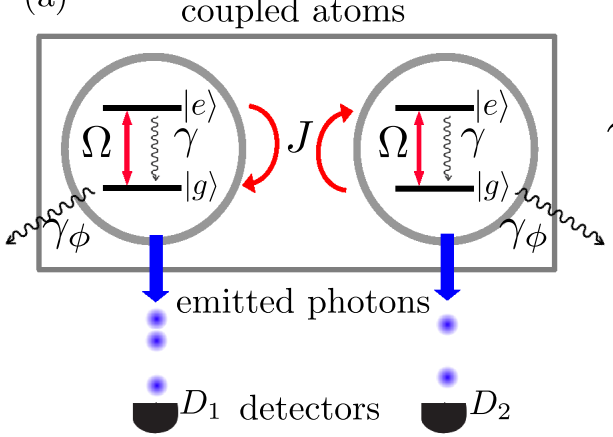

(c)

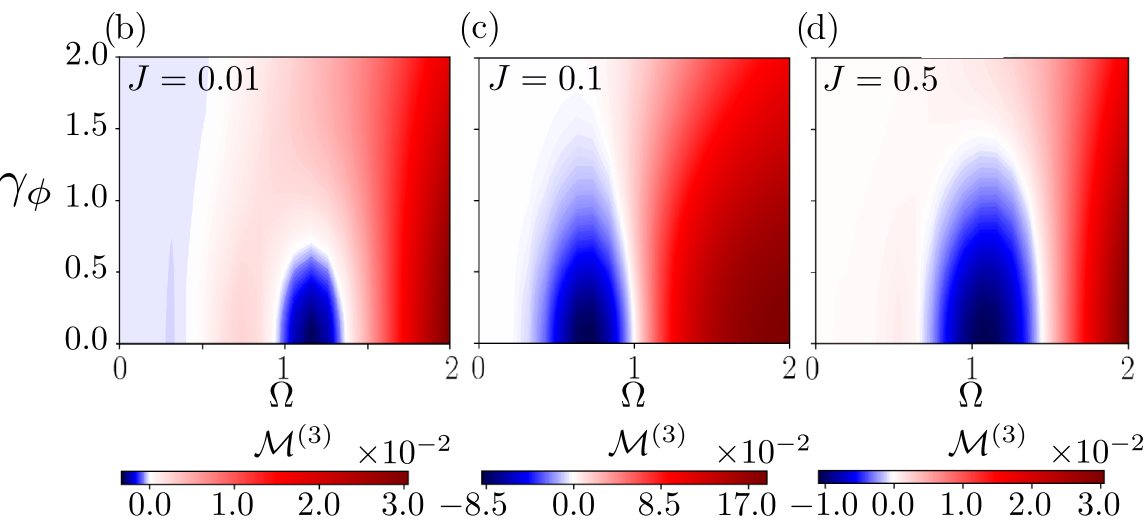

FIG. 2. Nonclassicality witness for emission from coupled atoms. (a) Third-order Vogel's determinant for a system of two coherently driven interacting atoms (coupling strength $J$ ) subject to dephasing, as a function of the dephasing rate $\gamma_{\phi}$ and the Rabi frequency $\Omega$. Panels (b)-(d) are for different coupling strengths: $J=0.01$ (b), $J=0.1$ (c), and $J=0.5$ (d). In all cases we observe a sharp separation between classical (positive Vogel's determinant) and quantum states of the emitted radiation (negative regions).

where the last equality follows from the optical equivalence theorem [33], $f\left(\alpha_{1}, \alpha_{2}\right)=\sum_{n, m=0}^{\infty} f_{n m} \hat{h}_{1}^{\dagger n}\left(\alpha_{1}, \alpha_{1}^{*}\right) \hat{h}_{2}^{m}\left(\alpha_{2}, \alpha_{2}^{*}\right)$, and where $\mathcal{P}\left(\alpha_{1}, \alpha_{2}\right)$ is the Glauber-Sudarshan distribution. Since $\left\langle: \hat{f}^{\dagger} \hat{f}:\right\rangle<0$ entails $\mathcal{P}\left(\alpha_{1}, \alpha_{2}\right)<0$ for some points $\left(\alpha_{1}, \alpha_{2}\right)$ of the phase space, the negativity of Eq. (6) is a clear signature of nonclassicality in radiation fields. Note that Eq. (6) is a quadratic form and is non-negative iff all the principal minors of the matrix $\mathcal{M}_{n m, k l}=\left\langle\hat{h}_{1}^{n+k} \hat{h}_{2}^{m+l}\right\rangle$ [see Eq. (A1) in Appendix] are positive, according to the Sylvester criterion [34]. Referring to the setup in Fig. 1 and according to Refs. [29,34], we express the VC in terms of click-counting operators, which, from the open quantum system point of view, take the form $\hat{h}_{j}=\hat{L}_{j}^{\dagger} \hat{L}_{j}$. Thus the elements of $\mathcal{M}_{n m, k l}$ are the moments of the photon-counting stationary distribution $P\left(K_{1}, K_{2}\right)$, which gives the probability to record $K_{1}$ clicks at photodetector $D_{1}$ and $K_{2}$ clicks at $D_{2}$. Hence, the criterion is now formulated in terms of time-integrated functions, like the photocurrents defined in Eq. (2). The moments in $\mathcal{M}_{n m, k l}$ are easily calculated through iterative derivation of the twomode moment-generating function associated with $P\left(K_{1}, K_{2}\right)$. Note that the mixed derivatives of the double-biased scaled cumulant-generating function $\theta\left(s_{1}, s_{2}\right)$ give us the mixed scaled cumulants directly linked to the two-mode moments in $\mathcal{M}_{n m, k l}$.

Different setups have been proposed, realized, and successfully used [20-22] in order to measure the click-counting distribution, thus uncovering quantum correlations of radiation fields. The click-counting distribution can approximate $P\left(K_{1}, K_{2}\right)$ involving photon-counting via a long-time measurement through photon-number-resolving detectors. As shown in Ref. [34], once the estimate of the stationary probabilities are known it is clearly possible to recover the moments in $\mathcal{M}_{n m, k l}$. Usually, the higher the order of the moment we calculate, the less accurate our estimate will be. In the cases we study next, low-order moments are enough to determine nonclassical features of radiation. It was shown [34] that the binomial form for the click-counting probability distribution holds for any positive-operator-valued measurement (POVM) either linear or nonlinear in the number of emitted photons. Thus the large-deviation formalism allows us to inherently access all the cumulants associated with any photon-counting process defined by the unraveling of the master equation.

\section{NONCLASSICALITY IN DISSIPATIVE CIRCUITS}

Typical coherent and squeezed radiation sources (pumped cavities, nonlinear active media) can be studied from the point of view of open quantum system theory [7]. Referring to the generic setup in Fig. 1, we now consider two different source structures: a pair of coupled two-level atoms, each coherently driven and subject to decay in their own emission channel, and two noninteracting atoms whose outputs are correlated via a beam splitter and a phase shifter. In both cases we introduce dephasing on each atom with the rate $\gamma_{\phi}$ : such a dephasing channel spoils coherence, hence it is expected to affect the nonclassicality of emitted light.

\section{TWO COUPLED ATOMS}

The total Hamiltonian of the system reads

$$
\begin{aligned}
\hat{H}= & \sum_{i=1}^{2}\left[\frac{\Omega}{2}\left(\hat{\sigma}_{i}^{+}+\hat{\sigma}_{i}^{-}\right)+\sqrt{\gamma}\left(\hat{\sigma}_{i}^{+} \hat{a}_{i}+\text { H.c. }\right)\right] \\
& +J\left(\hat{\sigma}_{1}^{+} \hat{\sigma}_{2}^{-}+\text {H.c. }\right),
\end{aligned}
$$

where $\gamma$ is the decay rate of each atom, $\Omega$ is the Rabi frequency, $\hat{\sigma}_{i}^{+}$and $\hat{\sigma}_{i}^{-}$are the ladder operators, $\hat{a}_{i}$ is the annihilation operator of the bosonic mode coupled to the $i$ th atom, ${ }^{1}$ and $J$ is the coupling strength. The jump operators of this elementary network are thus $\hat{J}_{1}=\sqrt{\gamma} \hat{\sigma}_{1}^{-}$and $\hat{J}_{2}=\sqrt{\gamma} \hat{\sigma}_{2}^{-}$. We can straightforwardly compute the large-deviation moments matrix and the corresponding Vogel determinants for the joint photon-counting probability distribution. It is worth noting that the second-order principal minor $\left(\mathcal{M}^{(2)}\right)$ does not contain information on the cross correlations between the emitted

\footnotetext{
${ }^{1}$ The $\hat{a}_{i}$ operators are intended as time-mode bosonic operators, or input modes, i.e., Fourier transform of field normal mode operators $\hat{a}_{\omega}$ under the assumption of white coupling between the system and the environment [35].
} 
field, which is our focus. Thus, it is necessary to consider the next-order minor. A numerical investigation of the thirdorder principal minor $\left(\mathcal{M}^{(3)}\right.$, see the Appendix) reveals the presence of quantum correlations between detection events in the emission channels. Figure 2 shows $\mathcal{M}^{(3)}$ as a function of the Rabi frequency $\Omega$ and the dephasing rate $\gamma_{\phi}$ for three values of the coupling rate $J$. In each case, nonclassicality is reduced as the dephasing rate grows. Negativity grows with $\Omega$, reaching a maximum and then saturating to a positive value. Dephasing destroys quantum coherences, making the atoms behave like classical objects, and this results in classical radiation fields, as expected. Higher values of $\Omega$ speed up Rabi oscillations: the effective coarse-graining time integration is lower bounded by $1 / \gamma$. Hence, we expect the time-integrated photocurrent becomes insensitive to the intensity fluctuations, resulting in a crossover between negative and non-negative values of the determinant. Furthermore we notice that the absolute minimum of the third-order determinant does not grow linearly with the coupling strength, but rather decreases when increasing $J$. It is indeed expected that the strong coupling between the two atoms makes the emission less likely to happen [36]. The strong-coupling contribution results in an effective shift of the energy level of the system and the perfect resonance condition is lost: the dominant component of the output fields becomes a vacuum, hence reducing the amount of cross correlations.

\section{NONINTERACTING ATOMS AND UNITARY CIRCUIT}

We consider next the case in which correlations can arise by processing the emitted fields of two noninteracting atoms $(J=0)$ through a unitary transformation employing a beam splitter $\left(\hat{\mathcal{U}}_{\mathrm{BS}}=\cos \zeta \mathbb{1}+i \sin \zeta \hat{\sigma}_{x}\right)$ and a phase shifter (Fig. 3). The corresponding jump operators read $\hat{J}_{1}=$ $\sqrt{\gamma_{1}} \cos \zeta \hat{\sigma}_{1}^{-}+i \sqrt{\gamma_{2}} \sin \zeta \hat{\sigma}_{2}^{-}$and $\hat{J}_{2}=i \sqrt{\gamma_{1}} \sin \zeta \hat{\sigma}_{1}^{-}+$ $\sqrt{\gamma_{2}} \cos \zeta \hat{\sigma}_{2}^{-}$. We set $\Omega=0.5 \gamma$ and $\gamma_{\phi}=0.1$ and study nonclassicality as a function of the reflectivity $R=\sin ^{2} \zeta$ and the phase difference $\delta$ between the two channels due to the phase shifter. For total transmission $(\zeta=0)$ and total reflection $(\zeta=\pi / 2)$, we notice that the determinant is positive. The maximum negativity is reached for a $50 / 50$ beam splitter and decreases as the phase shift $\delta$ grows. Thus, by adjusting appropriately the parameters of the optical circuit, such as the relative phase shift $\delta$, it is possible to enhance or destroy quantum interference effects of the output state.

\section{CONCLUSIONS}

In summary, we have shown how to detect signatures of nonclassicality through the statistics of time-integrated quantities, such as the photon counts. This offers the possibility to benchmark approaches for producing quantum resources for information and computation via general optical circuits and open quantum systems. Our findings can be extended both to imperfect detection and to recently proposed high-performing photon-number-resolving detection schemes [37]. Finally, we point out here a possible outlook of this work: the formalism here developed can be implemented to tackle the problem of characterizing many-body phases of matter by analyzing the statistical properties of emitted and scattered photons or bath quanta in general.
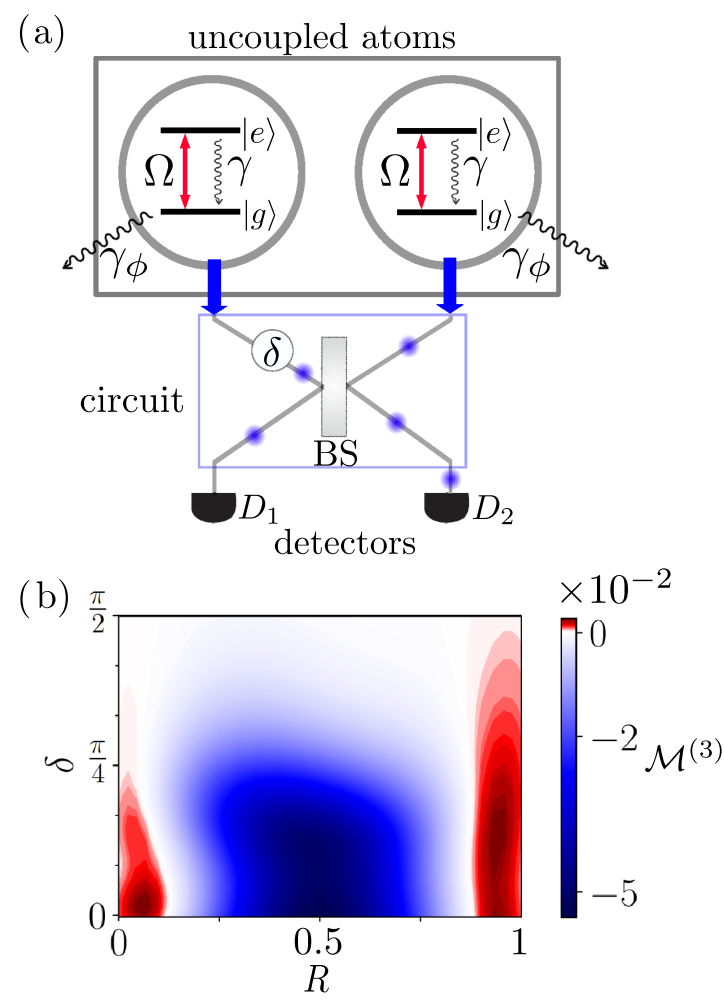

FIG. 3. Nonclassicality witness for emission from the optical circuit. The system (a) is composed of a two coherently driven noninteracting atoms subject to dephasing $\gamma_{\phi}$, emitting into the input channel of a generic unitary circuit composed of a phase shifter and a beam splitter. (b) We show the value of the third-order Vogel determinant as a function of the reflectivity $R=\sin ^{2} \zeta$ and the phase shift $\delta$.

\section{ACKNOWLEDGMENTS}

We thank Fabio Sciarrino, Taira Giordani, Fulvio Flamini, Iris Agresti, and Alessia Castellini for fruitful discussions. D.C. acknowledges the University of Nottingham for the hospitality. The research leading to these results has received funding from the European Union's H2020 research and innovation programme [Grant No. 800942 (ErBeStA)]. We acknowledge support under PRIN Project 2017SRN-BRK QUSHIP funded by MIUR.

\section{APPENDIX}

In line with Ref. [29] we put the elements $\mathcal{M}_{n m, k l}$ in ascending order with respect to the sum of the couples of indexes $(n+m)$ and such that $(n, m)<(n-1, m+1)$. The resulting matrix reads as follows:

$$
\mathcal{M}=\left(\begin{array}{cccccc}
1 & \left\langle\hat{h}_{1}\right\rangle & \left\langle\hat{h}_{2}\right\rangle & \left\langle\hat{h}_{1}^{2}\right\rangle & \left\langle\hat{h}_{1} \hat{h}_{2}\right\rangle & \ldots \\
\left\langle\hat{h}_{1}\right\rangle & \left\langle\hat{h}_{1}^{2}\right\rangle & \left\langle\hat{h}_{1} \hat{h}_{2}\right\rangle & \left\langle\hat{h}_{1}^{3}\right\rangle & \left\langle\hat{h}_{1}^{2} \hat{h}_{2}\right\rangle & \ldots \\
\left\langle\hat{h}_{2}\right\rangle & \left\langle\hat{h}_{1} \hat{h}_{2}\right\rangle & \left\langle\hat{h}_{2}^{2}\right\rangle & \left\langle\hat{h}_{1}^{2} \hat{h}_{2}\right\rangle & \left\langle\hat{h}_{1} \hat{h}_{2}^{2}\right\rangle & \ldots \\
\vdots & \vdots & \vdots & \vdots & \vdots &
\end{array}\right),
$$

where each element is a moment of the bivariate counting probability distribution associated with the counting 
operators. In the case of large deviation calculation, our average includes an integration over the duration time of trajectories [as in Eq. (2)]; thus we have direct access to the scaled time-integrated cumulant of such photon-counting probability distribution. The moments resulting by combination of scaled cumulants will be scaled in turn. From Eq. (3) we have that $\mathcal{M}_{n m, k l}=\frac{\partial^{n+k}}{\partial s_{1}^{n+k}} \frac{\partial^{m+l}}{\partial s_{2}^{m+l}} Z_{t}\left(s_{1}, s_{2}\right) \propto t^{n+m+k+l}$; i.e., the power of $t$ depends only on the position of the element in the matrix. Thus the power function of $t$ multiplying each summand featured in the determinant is invariant under permutation of indexes, the scaling resulting only in an overall positive factor $t^{f(N)}$ multiplying each $N$ th order Vogel determinant. Up to a multiplicative constant, the third-order principal minor we used in the examples reads as follows ( $s$ dependencies are omitted):

$$
\begin{aligned}
\mathcal{M}^{(3)}= & 2\left(\frac{\partial \theta}{\partial s_{1}} \frac{\partial \theta}{\partial s_{2}}\right)\left(\frac{\partial^{2} \theta}{\partial s_{1} \partial s_{2}}+\frac{\partial \theta}{\partial s_{1}} \frac{\partial \theta}{\partial s_{2}}\right) \\
& -\left(\frac{\partial^{2} \theta}{\partial s_{1} \partial s_{2}}+\frac{\partial \theta}{\partial s_{1}} \frac{\partial \theta}{\partial s_{2}}\right)^{2}-\left(\frac{\partial \theta}{\partial s_{1}}\right)^{2}\left[\frac{\partial^{2} \theta}{\partial s_{1} \partial s_{2}}\right. \\
& \left.+\left(\frac{\partial \theta}{\partial s_{2}}\right)^{2}\right]-\left(\frac{\partial \theta}{\partial s_{2}}\right)^{2}\left[\frac{\partial^{2} \theta}{\partial^{2} s_{1}}+\left(\frac{\partial \theta}{\partial s_{1}}\right)^{2}\right] \\
& +\left[\frac{\partial^{2} \theta}{\partial s_{1} \partial s_{2}}+\left(\frac{\partial \theta}{\partial s_{2}}\right)^{2}\right]\left[\frac{\partial^{2} \theta}{\partial s_{1}^{2}}+\left(\frac{\partial \theta}{\partial s_{1}}\right)^{2}\right]
\end{aligned}
$$

evaluated at point $s_{1}=s_{2}=0$.
[1] R. P. Feynman, Int. J. Theor. Phys. 21, 467 (1982).

[2] D. Loss and D. P. DiVincenzo, Phys. Rev. A 57, 120 (1998).

[3] A. Blais, R.-S. Huang, A. Wallraff, S. M. Girvin, and R. J. Schoelkopf, Phys. Rev. A 69, 062320 (2004).

[4] J. Preskill, Lecture Notes for Physics 229: Quantum Information and Computation, Vol. 16 (California Institute of Technology, 1998).

[5] A. Mari and J. Eisert, Phys. Rev. Lett. 109, 230503 (2012).

[6] G. M. Paule, Thermodynamics and Synchronization in Open Quantum Systems (Springer, Berlin, 2018), pp. 233-254.

[7] H. Carmichael, An Open Systems Approach to Quantum Optics: Lectures Presented at the Université Libre de Bruxelles, October 28 to November 4, 1991, Vol. 18 (Springer, Berlin, 2009).

[8] P. Kok, W. J. Munro, K. Nemoto, T. C. Ralph, J. P. Dowling, and G. J. Milburn, Rev. Mod. Phys. 79, 135 (2007).

[9] R. Ellis, Insur. Math. Econ. 17, 232 (1996).

[10] H. Touchette, Phys. Rep. 478, 1 (2009).

[11] A. Vulpiani, F. Cecconi, M. Cencini, A. Puglisi, and D. Vergni, The Legacy of the Law of Large Numbers (Springer, Berlin, 2014).

[12] D. Manzano and P. I. Hurtado, Phys. Rev. B 90, 125138 (2014).

[13] J. P. Garrahan and I. Lesanovsky, Phys. Rev. Lett. 104, 160601 (2010).

[14] J. P. Garrahan, A. D. Armour, and I. Lesanovsky, Phys. Rev. E 84, 021115 (2011).

[15] G. Buonaiuto, R. Jones, B. Olmos, and I. Lesanovsky, New J. Phys. 21, 113021 (2019).

[16] D. Cilluffo, S. Lorenzo, G. M. Palma, and F. Ciccarello, J. Stat. Mech.: Theory Exp. (2019) 104004.

[17] J. Sperling, M. Bohmann, W. Vogel, G. Harder, B. Brecht, V. Ansari, and C. Silberhorn, Phys. Rev. Lett. 115, 023601 (2015).

[18] G. Harder, T. J. Bartley, A. E. Lita, S. W. Nam, T. Gerrits, and C. Silberhorn, Phys. Rev. Lett. 116, 143601 (2016).

[19] J. Sperling, T. J. Bartley, G. Donati, M. Barbieri, X.-M. Jin, A. Datta, W. Vogel, and I. A. Walmsley, Phys. Rev. Lett. 117, 083601 (2016).
[20] G. Harder, C. Silberhorn, J. Rehacek, Z. Hradil, L. Motka, B. Stoklasa, and L. L. Sánchez-Soto, Phys. Rev. A 90, 042105 (2014).

[21] M. Avenhaus, K. Laiho, M. V. Chekhova, and C. Silberhorn, Phys. Rev. Lett. 104, 063602 (2010).

[22] J. Peřina, Jr., I. I. Arkhipov, V. Michálek, and O. Haderka, Phys. Rev. A 96, 043845 (2017).

[23] S. Haroche and J.-M. Raimond, Exploring the Quantum: Atoms, Cavities, and Photons (Oxford University, Oxford, 2006).

[24] G. Lindblad, Commun. Math. Phys. 48, 119 (1976).

[25] V. Gorini, A. Kossakowski, and E. C. G. Sudarshan, J. Math. Phys. 17, 821 (1976).

[26] P. Zoller, M. Marte, and D. F. Walls, Phys. Rev. A 35, 198 (1987).

[27] J. P. Garrahan, Physica A (Amsterdam, Neth.) 504, 130 (2018).

[28] C. Jordan and K. Jordán, Calculus of Finite Differences, Vol. 33 (American Mathematical Soc., 1965).

[29] E. V. Shchukin and W. Vogel, Phys. Rev. A 72, 043808 (2005).

[30] E. Shchukin, T. Richter, and W. Vogel, Phys. Rev. A 71, 011802(R) (2005).

[31] R. J. Glauber, Phys. Rev. 130, 2529 (1963).

[32] K. E. Cahill and R. J. Glauber, Phy. Rev. 177, 1857 (1969).

[33] W. P. Schleich, Quantum Optics in Phase Space (Wiley \& Sons, New York, 2011).

[34] J. Sperling, W. Vogel, and G. S. Agarwal, Phys. Rev. A 88, 043821 (2013).

[35] C. Gardiner, P. Zoller, and P. Zoller, Quantum Noise: A Handbook of Markovian and Non-Markovian Quantum Stochastic Methods with Applications to Quantum Optics (Springer, Berlin, 2004).

[36] M. Bamba, A. Imamoglu, I. Carusotto, and C. Ciuti, Phys. Rev. A 83, 021802(R) (2011).

[37] D. Malz and J. I. Cirac, arXiv:1906.12296. 